\title{
Long non-coding RNA LINC00238 suppresses the malignant phenotype of liver cancer by sponging miR-522
}

\author{
HONG-GANG QIAN ${ }^{1}$, QIONG WU ${ }^{2,3}$, JIAN-HUI WU $^{1}$, XIU-YUN TIAN $^{1}$, WEI XU $^{1}$ and CHUN-YI HAO ${ }^{1}$ \\ ${ }^{1}$ Key Laboratory of Carcinogenesis and Translational Research (Ministry of Education), Department of \\ Hepato-Pancreato-Biliary Surgery, Peking University Cancer Hospital and Institute, Beijing 100142; \\ ${ }^{2}$ MOE Key Lab, Bioinformatics, School of Life Sciences; ${ }^{3}$ Center for Synthetic and \\ Systems Biology, Tsinghua University, Beijing 100084, P.R. China
}

Received October 29, 2021; Accepted December 13, 2021

DOI: $10.3892 / \mathrm{mmr} .2022 .12587$

\begin{abstract}
Long non-coding RNAs can regulate the malignant tumor phenotype either as tumor suppressors or oncogenes. The present study investigated the underlying mechanism of LINC00238 in liver cancer. LINC00238 was identified as a downregulated molecule in The Cancer Genome Atlas liver hepatocellular carcinoma dataset through Gene Expression Profiling Interactive Analysis software. Through gain- and loss-of-function experiments, LINC00238 was confirmed as a tumor suppressor that could not only decrease cell viability, migration and invasion in vitro, but also tumorigenesis and tumor metastasis in vivo. By cytoplasmic and nuclear RNA isolation, LINC00238 was confirmed to be predominantly cytoplasmic. Mechanistically, RNA pull-down assays showed that LINC00238 sponged microRNA (miR)-522 and then reversed the inhibitory effects on two downstream targets, secreted frizzled related protein 2 and dickkopf1. Collectively, LINC00238 was identified as a tumor suppressor that acts via sponging miR-522 followed by silencing of downstream targets, suggesting that LINC00238 may have a key role in suppressing the malignant phenotype of liver cancer cells.
\end{abstract}

Correspondence to: Professor Hong-Gang Qian or Professor Chun-Yi Hao, Key Laboratory of Carcinogenesis and Translational Research (Ministry of Education), Department of Hepato-Pancreato-Biliary Surgery, Peking University Cancer Hospital and Institute, 52 Fucheng Road, Beijing 100142, P.R. China E-mail: qianhg@bjcancer.org

E-mail:dr.cyhao@gmail.com

Abbreviations: lncRNA, long non-coding RNA; TCGA, The Cancer Genome Atlas; LIHC, liver hepatocellular carcinoma; GEPIA, Gene Expression Profiling Interactive Analysis; RT-qPCR, reverse transcription-quantitative PCR; CCK-8, Cell Counting Kit-8; SFRP2, secreted frizzled related protein 2; DKK1, dickkopf 1

Key words: IncRNA, LINC00238, miR-522, SFRP2, DKK1, liver cancer

\section{Introduction}

Liver cancer is the fifth most frequent fatal malignancy in the United States (1), but ranks second in China (2) due to the high risk of factors, such as hepatitis B virus (HBV) or hepatitis $\mathrm{C}$ virus (HCV) infection, fatty liver disease and alcohol-related cirrhosis. In total 5-15\% of patients are suitable for surgical removal, which is only for early-stage patients (3). The prognosis for unresectable stage liver cancer is very poor, with a median survival time of $6-20$ months and $<5 \%$ for the 5 -year survival rate (4). Therefore, discovering prognostic or therapeutic biomarkers is urgently required for patients with liver cancer.

Long non-coding RNAs (lncRNAs) are defined as non-coding RNAs $>200$ nucleotides (5). Although lncRNAs undergo similar processing as mRNAs, including splicing, capping, polyadenylation and editing, they lack significant open reading frames (5). Increasing evidence suggests that lncRNAs participate in every aspect of the life cycle of a gene, including transcription, splicing, RNA decay and translation (6). Cytoplasmic lncRNAs can regulate gene expression via diverse mechanisms, either by sponging microRNAs (miRNAs/miRs) or altering mRNA stability (7).

To date, a total of 74 deregulated liver cancer-associated lncRNAs have been reported, with 52 upregulated lncRNAs exhibiting oncogenic properties and 22 downregulated lncRNAs exhibiting tumor-suppressive properties (8). Therefore, these insights reveal that novel lncRNAs may be potential biomarkers and enable the design of precision therapy for liver cancer. Previously, it was reported that HBV infection inhibited the expression of LINC00238, which was found to be significantly downregulated in HBV-positive liver tissues, HBV-expressing cell lines and HBV transient-expressing cells (9). Furthermore, overexpression of LINC00238 could suppress HBV replication (9). Chronic HBV infection is a major risk factor for liver cancer, and notably, in China, HBV-related liver cancer accounts for $\sim 85 \%$ of liver cancer cases due to the high prevalence of HBV infection (10). However, to the best of our knowledge, the role of LINC00238 in liver cancer progression is still unclear.

Gene Expression Profiling Interactive Analysis (GEPIA) is a web-based tool that can deliver fast and customizable functionalities based on The Cancer Genome Atlas (TCGA) and 
The Genotype-Tissue Expression data (11). Researchers can perform comprehensive expression analyses through GEPIA by customizable functions, including differential expression analysis, profiling plotting, correlation analysis, patient survival analysis, similar gene detection and dimensionality reduction analysis (11). The chick embryo chorioallantoic membrane (CAM) assay is an alternative in vivo experimental model suitable for cancer studies (12). Compared with the mouse xenograft model, the CAM is a low cost method that is naturally immune-incompetent, favorable to tumor grafting, and enables the ability of perform neovascularization and invasion assays to evaluate the ability of cells to be tumorigenic and invade and metastasize into the embryo (13). It is widely used for the investigation of multiple steps of tumor progression, metastatic behavior and molecular deregulated pathways, including the evaluation of stem cell activity in breast cancer (13), sarcoma $(14,15)$, lymphoma (16), liver cancer (17) and melanoma (18).

In the present study, data mining was performed by GEPIA analysis of IncRNAs for liver hepatocellular carcinoma [LIHC (TCGA-LIHC)], which led to the detection of the expression profile of LINC00238 in liver cancer, and identified it as a tumor suppressor. Mechanistically, LINC00238 acted as a molecular sponge to adsorb miR-522, resulting in the reversal of the inhibitory effects on two downstream targets, secreted frizzled related protein 2 (SFRP2) and dickkopf1 (DKK1). Therefore, the functions of a novel lncRNA that regulated the malignant phenotype of liver cancer was described.

\section{Materials and methods}

GEPIA analysis of TCGA and Gene Expression Omnibus (GEO) data. GEPIA (11) (http://gepia.cancer-pku.cn) was used to analyze the aberrantly expressed lncRNAs by differential expression analysis of TCGA-LIHC. The downregulated lncRNAs among the differentially expressed genes (DEGs) with $\log _{2}$ fold change (FC) cutoff $=1$ and q-value cutoff $=0.01$ were selected. Next, Kaplan-Meier survival analysis was performed followed by a log-rank test for these downregulated lncRNAs to obtain lncRNAs associated with the overall survival of patients after surgery by the median expression level of each lncRNA in LIHC $(\mathrm{P}<0.1$, two-tailed test $\mathrm{P}<0.1$ means one-tailed test $\mathrm{P}<0.05$ because this study was only concerned about one direction, not both).

Cell line and cell culture. Two liver cancer cell lines (HepG2 and Huh7) and 293T cells were obtained from the National Infrastructure of Cell Line Resource. Cell lines were confirmed to be free of mycoplasma contamination by PCR and three short tandem repeat (STR) loci. Cells were cultured in RPMI-1640 (Gibco; Thermo Fisher Scientific, Inc.) supplemented with penicillin $(50 \mathrm{U} / \mathrm{ml})$, streptomycin $(50 \mathrm{~g} / \mathrm{ml})$ and $10 \%$ heat-inactivated fetal bovine serum (FBS, Thermo Fisher Scientific Inc.) in a humidified $5 \% \mathrm{CO}_{2}$ atmosphere at $37^{\circ} \mathrm{C}$.

In vivo tumor growth and metastasis. A modified chick embryo CAM assay was used to assess tumor growth and metastatic characteristics. Briefly, a total of 35 10-day-old SPF white leghorn chicken embryo eggs (Beijing Vital River Laboratory Animal Technology Co., Ltd.) were randomized into groups
( $n=5 /$ group). A square window was opened in the shell under aseptic conditions after sterilization with $75 \%$ ethanol. A total of $5 \times 10^{6}$ cells in $50 \mu \mathrm{l}$ PBS (including Huh7 control cells, LINC00238 overexpression Huh7 cells, scramble HepG2 cells, LINC00238 shRNA HepG2 cells, LINC00238 overexpression Huh7 cells transfected with NC and miR-522 mimics) were labeled with CM-DiI (red fluorescent dye) in 5\% glucose for $15 \mathrm{~min}$ at $37^{\circ} \mathrm{C}$ and inoculated onto each CAM. Eggs were resealed with sterilized tape and returned to a humidified $37^{\circ} \mathrm{C}$ incubator for an additional 7 days (before hatching out, normally chicken eggs would hatch in 21 days). The tumors that grew on each CAM of the 18-day-old chicken embryos were dissected and weighed. Then, the chicken embryos were sacrificed by cervical dislocation on day 18 after the incubation period began. The fresh lungs were isolated, flattened by two slides and evaluated under a fluorescence microscope (Leica Microsystems, Inc.) to track the distant metastatic tumor loci (with red fluorescence) (19).

RNA extraction and reverse transcription-quantitative (RT-q) PCR analysis. Total RNA was isolated from control or LINC00238 overexpression Huh7 cells, scramble or LINC00238-shRNA HepG2 cells, NC or miR-522 transfected Huh7 or LINC00238 overexpression Huh7 cells by using a miRNeasy mini kit (Qiagen, Inc.). Separation and purification of cytoplasmic and nuclear RNA from HepG2 and Huh7 cells was performed using a Cytoplasmic \& Nuclear RNA Purification kit (Norgen Biotek Corp.). IncLocator (csbio. sjtu.edu.cn/bioinf/lncLocator/) was used to predict the lncRNA subcellular location. First strand cDNA was synthesized from $5 \mu \mathrm{g}$ RNA using random primers and Moloney Murine Leukemia Virus reverse transcriptase (M-MLV RT; Invitrogen; Thermo Fisher Scientific, Inc.). For miRNA detection, $100 \mathrm{ng}$ of RNA was first added to polyA tails by polyA polymerase (NEB), and then CDNA was synthesized by OligodT-Adaptor and M-MLV RT according to the manufacturer's protocol. qPCR was performed using SYBR Green PCR Master Mix (Applied Biosystems; Thermo Fisher Scientific, Inc.) on an ABI 7500 System (Applied Biosystems; Thermo Fisher Scientific, Inc.). The thermocycling conditions were initial denaturation at $95^{\circ} \mathrm{C}$ for $5 \mathrm{~min}$, followed by 45 amplification cycles of $95^{\circ} \mathrm{C}$ for $10 \mathrm{sec}, 60^{\circ} \mathrm{C}$ for $20 \mathrm{sec}, 72^{\circ} \mathrm{C}$ for $42 \mathrm{sec}$ and a final extension $1 \mathrm{~min}$ at $72^{\circ} \mathrm{C}$. The gene expression level was calculated by the $2^{-\Delta \Delta \mathrm{Cq}}$ method (20), where $\Delta \mathrm{Cq}=\mathrm{Cq}$ (gene)-Cq (GAPDH). For lncRNA and miRNA expression, GAPDH and U6 were used as internal reference genes, respectively. qPCR data are represented as the mean \pm SD from three independent experiments. Sequences for primers are listed in Table SI. The primers for amplification of LINC00238 were designed in the common region of three isotypes of $\mathrm{V} 1, \mathrm{~V} 3$ and V4.

Plasmid construction and cell transfection. LINC00238 (NR_024338.3, isotype V1) was subcloned into a pcDNA3.1 (+) (Invitrogen; Thermo Fisher Scientific, Inc.) expression vector. A total of $10 \mu \mathrm{g}$ of blank vector or LINC00238 plasmids were transfected into $5 \times 10^{6}$ Huh7 cells for $48 \mathrm{~h}$ and selected with $500 \mathrm{mg} / \mathrm{ml} \mathrm{G} 418$ solution for 1 week to obtain control group and stable LINC00238 overexpression group maintained in $250 \mathrm{mg} / \mathrm{ml} \mathrm{G} 418$ solution. A total of $5 \mu \mathrm{g}$ of $3 \mathrm{rd}$ lentivirus RNAi 
shuttle vector (plenti6-U6, originally from Invitrogen plenti6 backbone) containing the sequence for scramble control or for the short hairpin RNA (shRNA) of LINC00238 was transfected into $1 \times 10^{6} 293 \mathrm{FT}$ cells together with the $9 \mu \mathrm{g}$ packaging plasmids containing PLP1, PLP2 and pLP/VSVG to generate lentiviruses for $48 \mathrm{~h}$ at $37^{\circ} \mathrm{C}$ to collect lentiviral particles in the supernatant by centrifuge at $1,000 \mathrm{x}$ g for $5 \mathrm{~min}$. Lentivirus at an MOI of 20 were added into HepG2 cells seeded in 6 -well plates (at a density of $5 \times 10^{4}$ cells/well), screened with $1 \mu \mathrm{g} / \mathrm{ml}$ blasticidine $\mathrm{S}$ and maintained in $0.5 \mu \mathrm{g} / \mathrm{ml}$ blasticidine $\mathrm{S}$ solution. All plasmids were confirmed by sequencing. Targeting sequences for shRNAs are listed in Table SI. For miRNA overexpression, $10 \mathrm{pmol} / \mathrm{ml}$ double-stranded miR-522 mimics (5'-aaaaugguucccuuuagagugu-3') and negative control (micrON mimic NC \#22) oligonucleotides were purchased from Guangzhou Ruibio Co., Ltd., and transfected into LINC00238 overexpression $1 \times 10^{6} \mathrm{Huh} 7$ cells by using Lipofectamine $^{\circledR} 2000$ (Invitrogen; Thermo Fisher Scientific, Inc.), according to the manufacturer's instructions. At $24 \mathrm{~h}$ post-transfection, subsequent experiments were performed.

Cell viability and plate colony formation assays. A total of $1 \times 10^{3}$ cells/well, including control or LINC00238 overexpression Huh7 cells, scramble or LINC00238-shRNA HepG2 cells, NC or miR-522 transfected Huh7 or LINC00238 overexpression Huh7 cells, were seeded into 96-well plates. Cell viability was evaluated by a Cell Counting Kit-8 (CCK-8; Dojindo Molecular Technologies, Inc.). Briefly, $10 \mu \mathrm{l} \mathrm{CCK-8}$ reagents were incubated with cells for $1 \mathrm{~h}$, and then the absorbance at $450 \mathrm{~nm}$ was measured with a microplate reader. For the plate colony formation assay, 500 cells/well, including control or LINC00238 overexpression Huh7 cells, scramble or LINC00238-shRNA HepG2 cells, NC or miR-522 transfected Huh7 or LINC00238 overexpression Huh7 cells, were cultured in 6-well plates for 1 week. Colonies were fixed in $4 \%$ formaldehyde for $5 \mathrm{~min}$ at room temperature and stained with $0.1 \%$ crystal violet for $10 \mathrm{~min}$ at room temperature. Colonies numbers were counted by Image $\mathbf{J}$ software.

Cell migration and invasion assays. Control or LINC00238 overexpression Huh7 cells, scramble or LINC00238-shRNA HepG2 cells, NC or miR-522 transfected Huh7 or LINC00238 overexpression Huh7 cells $\left(1 \times 10^{4}\right)$ pretreated with $10 \mu \mathrm{g} / \mathrm{ml}$ mitomycin-C (Sigma-Aldrich; Merck KGaA) for $1 \mathrm{~h}$ at $37^{\circ} \mathrm{C}$ were added to the upper chamber of a Transwell plate (for invasion assay, Transwell membrane were precoated with $50 \mu 1$ 1:40 diluted Matrigel for $1 \mathrm{~h}$ at $37^{\circ} \mathrm{C}$ ) with an $8.0-\mu \mathrm{m}$ pore polycarbonate membrane insert in $100 \mu \mathrm{l}$ RPMI-1640 containing 1\% FBS. A total of $500 \mu \mathrm{l}$ RPMI-1640 with $10 \%$ FBS was added to the lower chamber as a chemoattractant. After $24 \mathrm{~h}$ at $37^{\circ} \mathrm{C}$ in the cell incubator, cells were fixed for 5 min with $4 \%$ formaldehyde at room temperature and stained with $1 \%$ crystal violet for $1 \mathrm{~min}$ at room temperature. After removing the cells inside the membrane, the number of migrated and invasive cells was imaged in four randomly selected microscopic fields under light microscope (Leica).

Immunoblotting. Cells or tumor tissues were lysed with radioimmunoprecipitation assay (RIPA) buffer containing a protease inhibitor cocktail (Roche Diagnostics). The protein concentration was assessed with a BCA protein assay kit (Bio-Rad Laboratories, Inc.). Equal amounts of protein for each sample $(30 \mu \mathrm{g})$ were separated on $10 \%$ SDS-PAGE gels and subsequently transferred to PVDF membranes (Millipore Sigma). The membranes were blocked for $1 \mathrm{~h}$ in PBS containing 5\% non-fat milk at room temperature and then incubated overnight with SFRP2 (cat. no. \#4687, Cell Signaling Technology, Inc.), DKK1 (cat. no. ab109416, Abcam) and GAPDH (cat. no. \#5174, Cell Signaling Technology, Inc.) antibodies $(1: 5,000)$ at $4^{\circ} \mathrm{C}$. The membranes were then incubated with HRP-conjugated secondary antibodies (1:50,000, \#111-005-003, Jackson ImmunoResearch) at room temperature for $1 \mathrm{~h}$ and visualized with ECL detection reagents (Millipore Sigma). Images were captured by AI600 version 1.2.0 (GE Healthcare) on an Amersham Imager 600 (GE Healthcare).

RNA pull-down. For the in vivo pull-down assay, LINC00238-6xMS2bs plasmids in the pcDNA3.1 backbone (Invitrogen; Thermo Fisher Scientific, Inc.) containing six repeat MS2-binding site RNA sequences and MS2 expression plasmids with Flag tags in the pcDNA3.1 backbone (Invitrogen; Thermo Fisher Scientific, Inc.) were co-transfected into $1 \times 10^{6}$ 293T cells. After $48 \mathrm{~h}$ transfection cells were lysed by $500 \mu \mathrm{l}$ NP40 cell lysis buffer (Thermo Fisher Scientific, Inc.), then $500 \mu 1$ lysate were incubated with $50 \mu 1$ Anti-FLAG ${ }^{\circledR}$ M2 Magnetic Beads (\# M8823, Sigma-Aldrich; Merck KGaA) overnight at $4^{\circ} \mathrm{C}$. After washing with PBS three times using magnetic separation rack at room temperature, the pull-down product was eluted by $100 \mu \mathrm{l} 3 \mathrm{X} \mathrm{FLAG}^{\circledR}$ peptide. Biotin-based RNA pull-down assays were carried out according to the protocol (21). The pull-down RNAs were isolated, purified and cDNA was synthesized, followed by analysis via qPCR as aforementioned.

Reporter gene assay. Predicted consequential pairing of target region 'CCAUUU' and miR-522 were searched by Gene Runner software version 6.5.52 Beta (Frank Buquicchio and Michael Spruyt). The wild-type and mutant LINC00238 were incorporated into the pGL3-control vector (Promega Corporation) via the In-Fusion HD Cloning kit (Takara Bio). To determine the relative luciferase activity, $1 \times 10^{4} 293$ cells were seeded into 24-well plates and co-transfected with $500 \mathrm{ng}$ pGL3-control containing LINC00238 wild-type or mutant, 26 ng pRL-TK plasmid (Promega Corporation) expressing Renilla luciferase, and 20 pmol miR-522 mimics or NC using Lipofectamine 2000 $(\mathrm{n}=4)$. Firefly and Renilla luciferase activity in the cell lysates was measured $24 \mathrm{~h}$ after transfection using a dual-luciferase reporter assay kit (Promega Corporation). Firefly luciferase activity was normalized to that of Renilla luciferase for each sample.

Statistical analysis. Experiments were performed in triplicate. Data were analyzed with SPSS 26 (IBM Corp.) and GraphPad Prism 8 (GraphPad Software, Inc.). The continuous variables with normal distribution and equal variance ( $\mathrm{F}$ test) between/within the groups are expressed as the mean $\pm \mathrm{SD}$, and the statistical significance was determined using an unpaired two-tailed Student's t-test between two groups, one-way ANOVA followed by a Bonferroni post hoc test for 
A

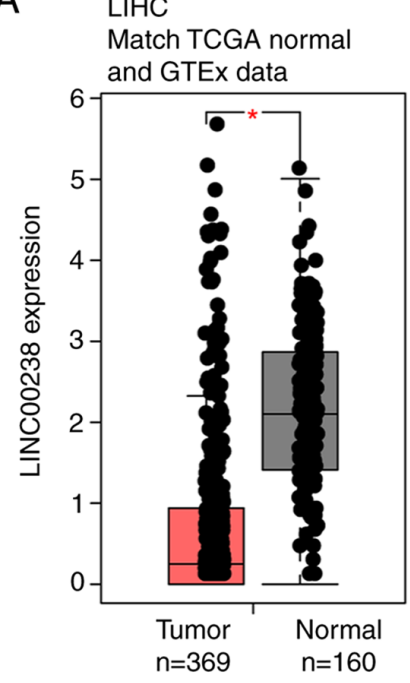

B

LIHC Match TCGA normal data

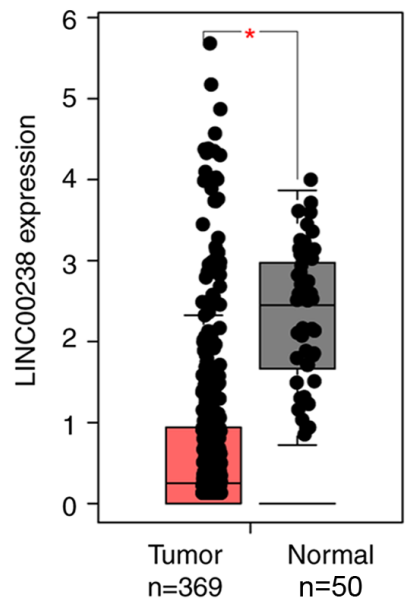

C
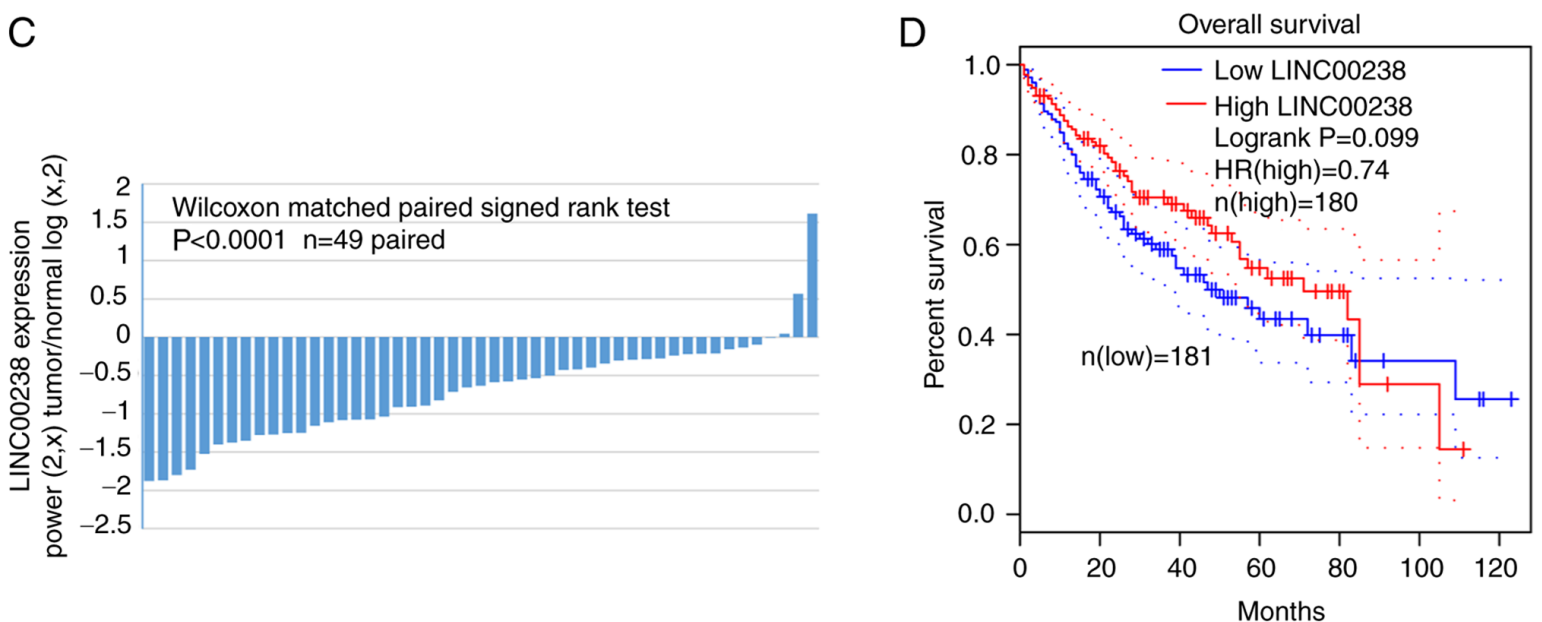

Figure 1. Low levels of LINC00238 expression are associated with poor prognosis of patients with liver cancer. (A,B) LINC00238 was downregulated in liver cancer tissues compared with in normal tissues from TCGA and Gene Expression Omnibus databases by Gene Expression Profiling Interactive Analysis. (C) The expression analysis showed that LINC00238 was downregulated in 46/49 hepatocellular carcinoma and matched normal tissues from TCGA database. (D) Kaplan-Meier analysis of overall survival from TCGA database revealed that patients with lower expression of LINC00238 in tumor tissues had poorer prognosis. * $\mathrm{P}<0.05$. TCGA, The Cancer Genome Atlas; LIHC, liver hepatocellular carcinoma; GTEx, Genotype-Tissue Expression.

multiple comparisons and two-way ANOVA for cell viability analysis. $\mathrm{P}<0.05$ was considered to indicate a statistically significant difference.

\section{Results}

Identification of the downregulated lncRNAs in liver cancer tissues. A total of 15 downregulated lncRNAs were screened out in liver cancer tissues by GEPIA $\left(\mathrm{FC}>2, \mathrm{P}_{\mathrm{adj}}<0.01\right.$, Table SII). Next, Kaplan-Meier survival analysis suggested that five lncRNAs (LINC01554, LINC01093, LINC01018, LINC01370 and LINC00238) were associated with the overall survival of patients after surgery by the median expression level of each lncRNA as cut-off value (Log-rank test $\mathrm{P}<0.1$, Table SII; Fig. 1D for LINC00238 and Fig. S1 for the remaining IncRNAs). LINC01554 (22,23), LINC01093 (24,25) and LINC01018 $(26,27)$ have been reported to have roles in liver cancer. LINC00238 has been reported to be associated with HBV infection (9), which accounts for $\sim 50 \%$ of liver cancer cases worldwide (28). Recently, LINC00238 was also reported as a tumor suppressor in liver cancer (29). Although Jiang reported that LINC00238 inhibited liver cancer progression by activating the TMEM106C-mediated apoptosis pathway through in vitro assays, further experiments are still required, including in vivo experiments on the subcellular location and other mechanisms involved (29). Thus, LINC00238 was selected for further study. As shown in Fig. 1A and B, the expression of LINC00238 was significantly decreased in liver cancer tissues compared with normal tissues according to the GEPIA website. In addition, as indicated in Fig. 1C, LINC00238 was downregulated in $91.8 \%(45 / 49, \mathrm{P}<0.0001)$ of liver cancer tissues by paired Student's t-test analysis of matched and normal tissues from TCGA dataset. K-M survival curves indicated that patients with high LINC00238 expression showed longer overall survival times (median survival 80 months vs. 50 months; hazard ratio, 0.74; $\mathrm{P}=0.099$; Fig. 1D).

LINC00238 overexpression impairs the liver cancer malignant phenotype in vitro. LINC00238 expression in human liver cancer cells, including HepG2 and Huh7 cells, 

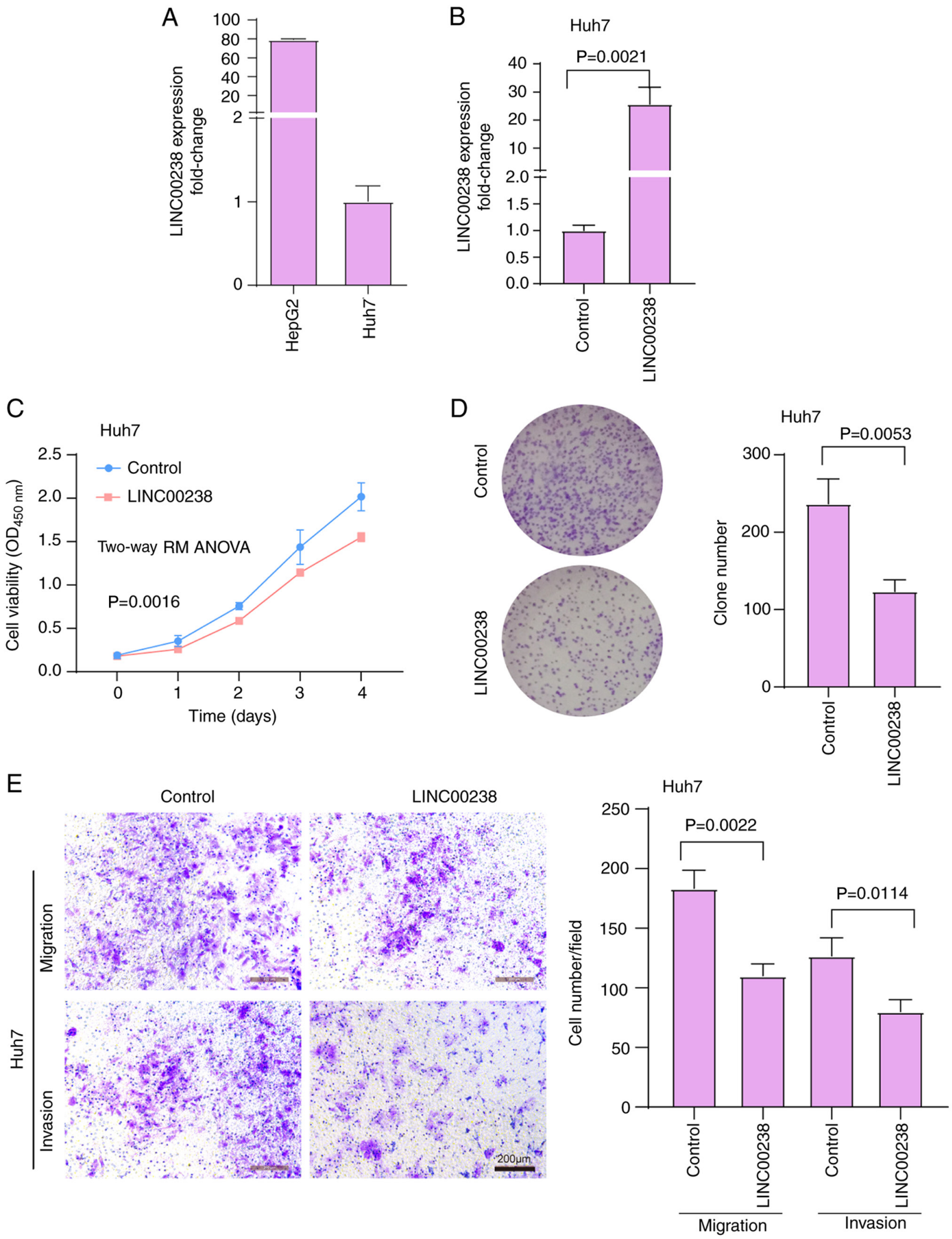

Figure 2. Overexpression of LINC00238 inhibits liver cancer cell viability, invasion and migration in vitro. (A,B) Expression levels of LINC00238 in cells were determined via reverse transcription-quantitative PCR. (C) Cell Counting Kit-8 assays indicated that overexpression of LINC00238 suppressed cell viability. (D) Plate colony formation assays showed that overexpression of LINC00238 suppressed cell colony formation. (E) Transwell assays suggested that overexpression of LINC00238 reduced cell migration and invasion. The number of cells was counted in four different fields. Scale bar, $200 \mu \mathrm{m}$.

was then quantified. The relative expression of LINC00238 in Huh7 cells was lower than that in HepG2 cells (Fig. 2A). Huh7 cells (relatively low LINC00238 expression) were chosen for the overexpression experiments and HepG2 cells (relatively high LINC00238 expression) were chosen for the knockdown experiments. Compared with control cells, LINC00238 expression was increased by 24 -fold after overexpression, as detected by qPCR (Fig. 2B). Overexpression of LINC00238 


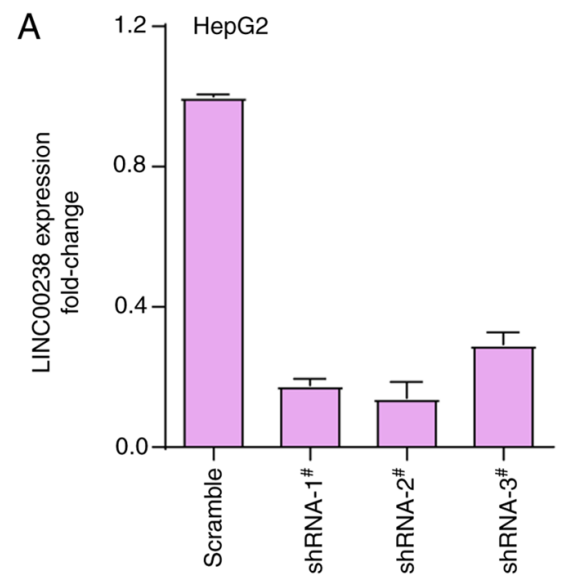

C
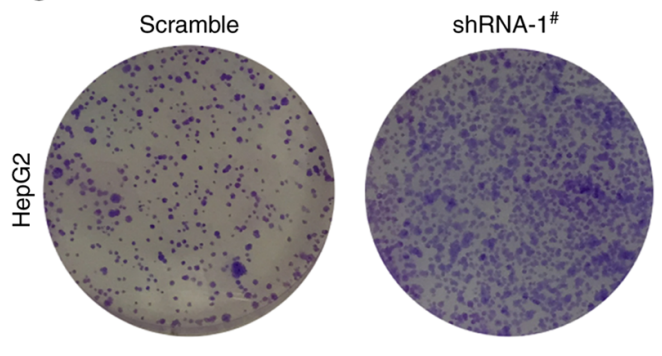

B

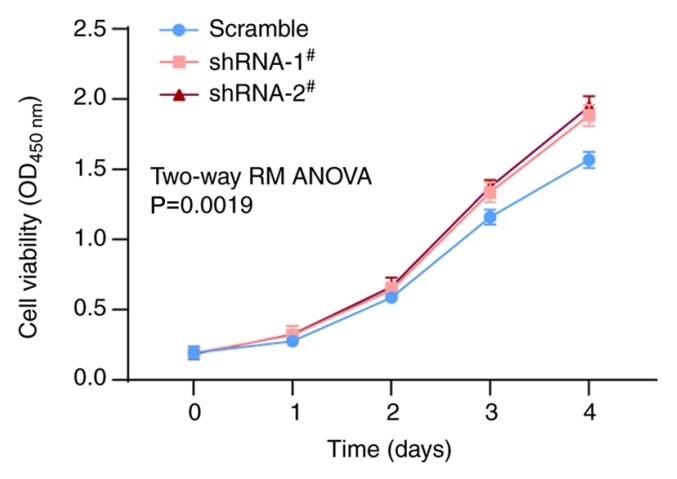

HepG2
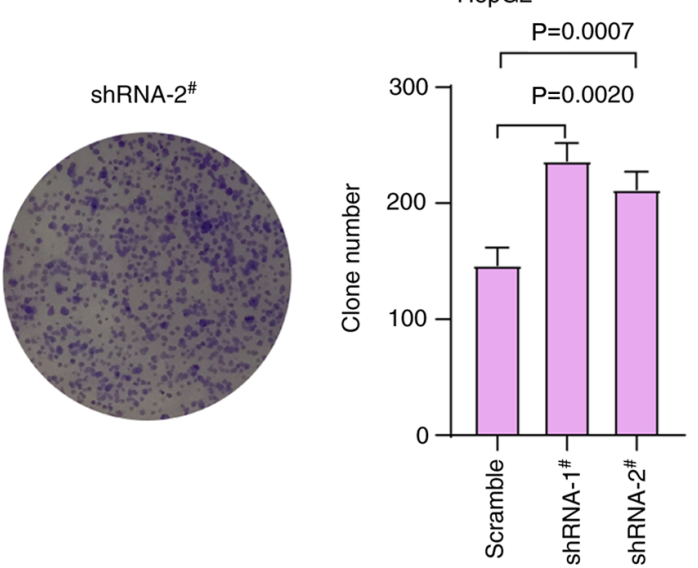

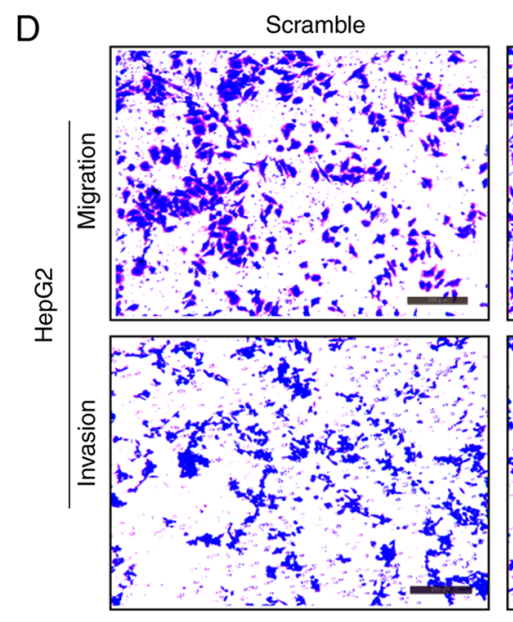

shRNA-1 ${ }^{\#}$
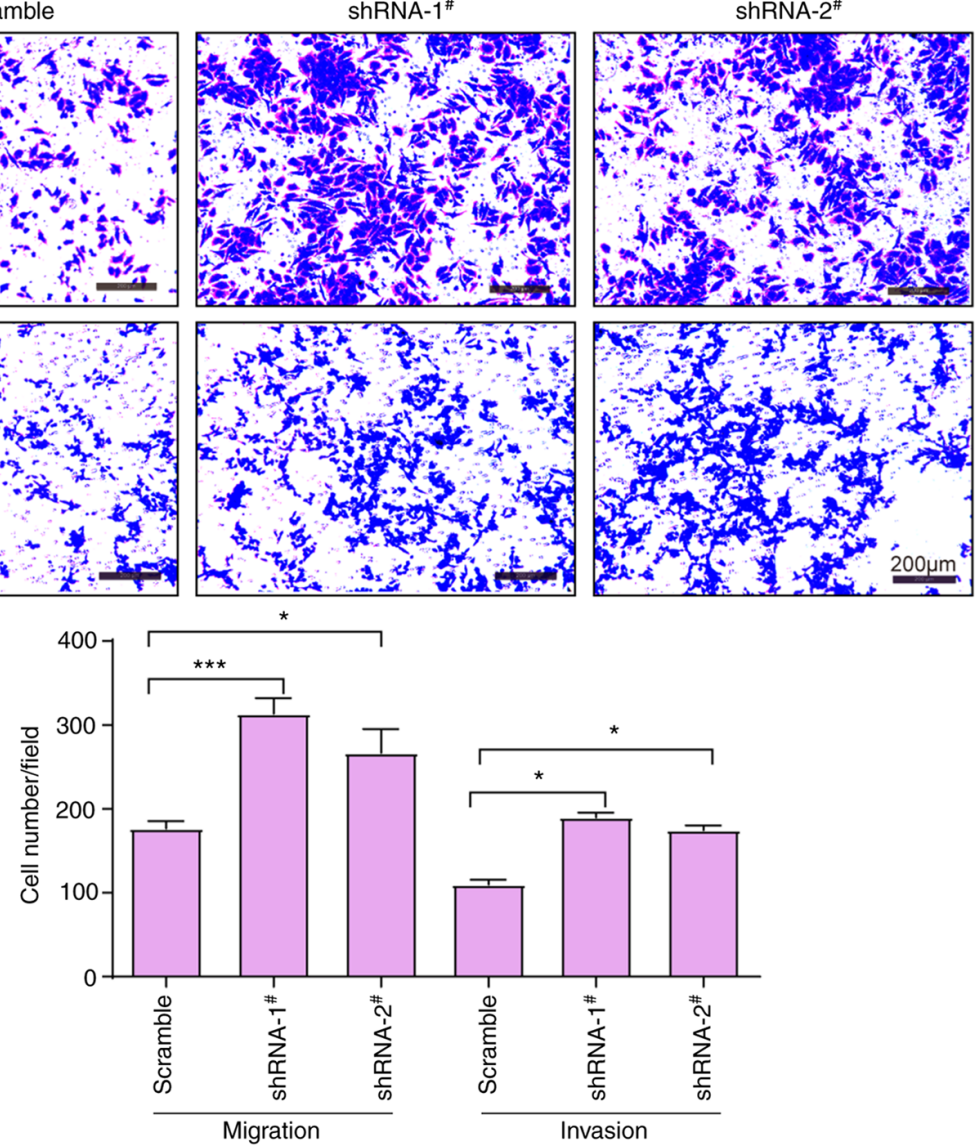

Figure 3. Knockdown of LINC00238 promotes liver cancer cell viability, invasion and migration in vitro. (A) Expression of LINC00238 in HepG2 cells after shRNA-mediated knockdown of LINC00238 was detected via reverse transcription-quantitative PCR. (B) Cell Counting Kit- 8 assays suggested that the knockdown of LINC00238 promoted cell viability. (C) Plate colony formation assays indicated that the knockdown of LINC00238 enhanced cell colony formation. (D) Transwell assays showed that the knockdown of LINC00238 promoted cell migration and invasion. The number of cells was counted in four different fields. Scale bar, $200 \mu \mathrm{m} .{ }^{*} \mathrm{P}<0.05,{ }^{* * * *} \mathrm{P}<0.001$. shRNA, short hairpin RNA. 
A
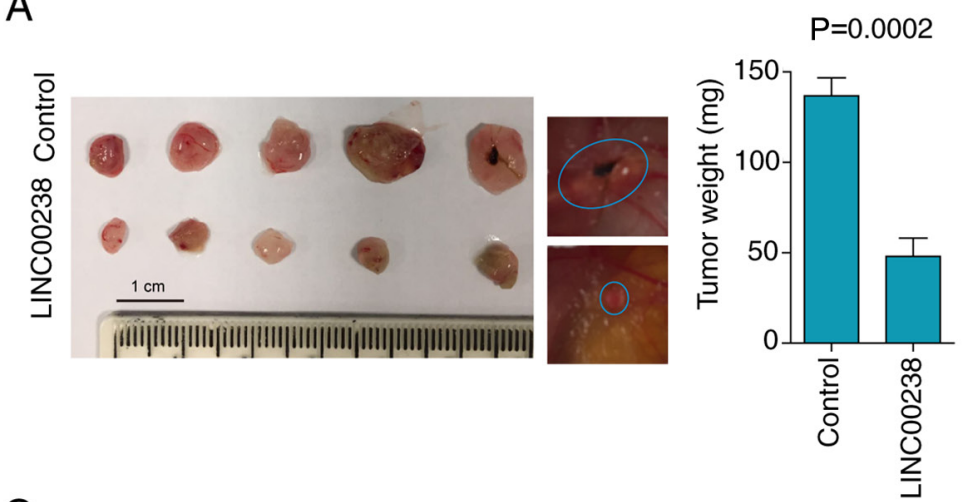

C

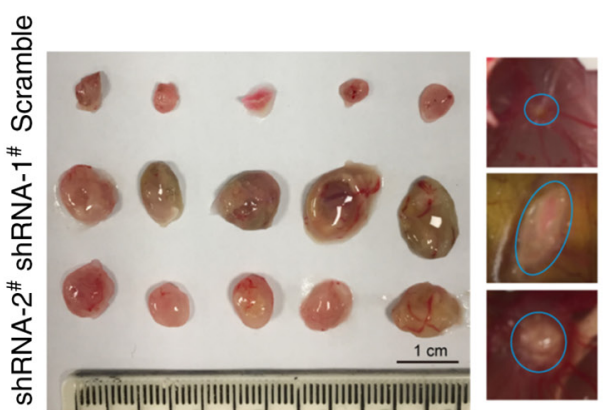

B
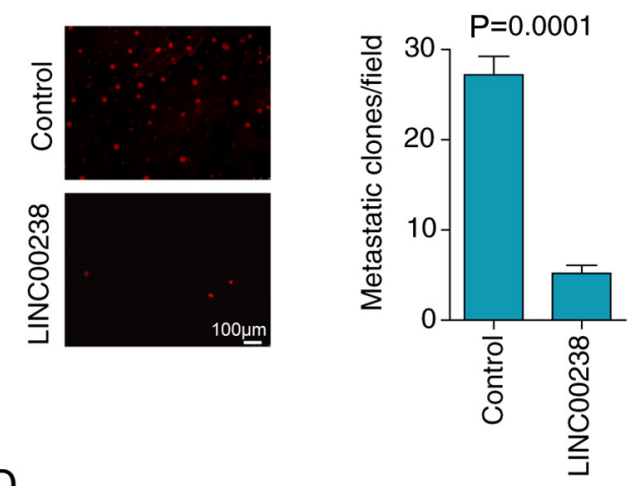

$\mathrm{D}$
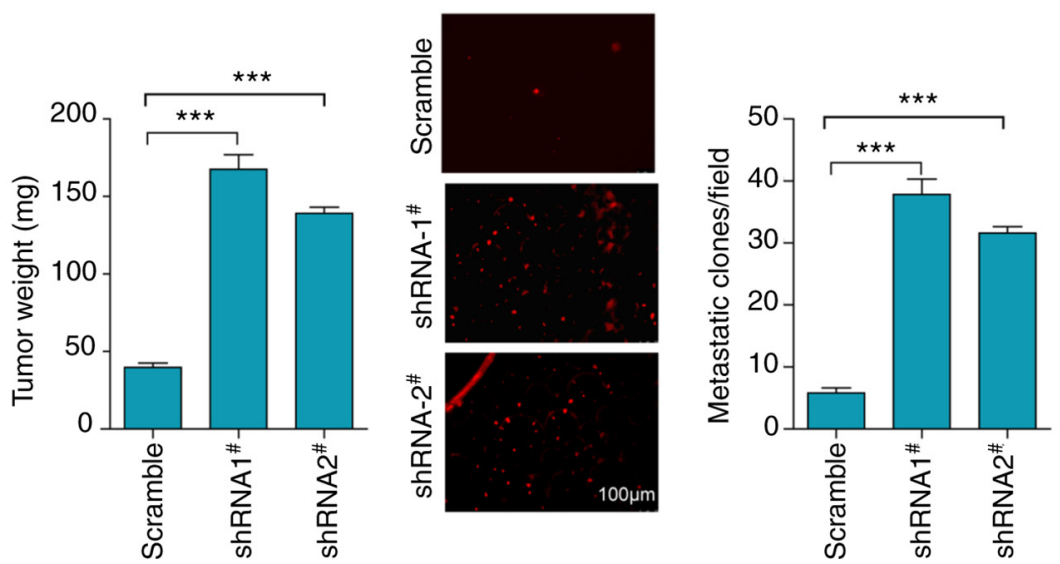

Figure 4. LINC00238 reduces tumor growth and progression in vivo. Compared with control cells, LINC00238-overexpressing Huh7 cells not only initiated (A) smaller and lighter tumors on the CAM as evaluated based on the tumor weight of each group, but also (B) showed fewer lung metastasis lesions as evaluated by the number of metastatic lesions under a fluorescence microscope. Compared with scramble cells, LINC00238-knockdown HepG2 cells not only initiated (C) larger and heavier tumors on the CAM, but also (D) showed more lung metastasis lesions. The data in the bar graphs were calculated as the mean \pm SD for each group. ${ }^{* * *} \mathrm{P}<0.001$. CAM, chorioallantoic membrane; shRNA, short hairpin RNA.

reduced Huh7 cell viability (Fig. 2C) and colony formation ( $47.9 \%$ inhibition; Fig. 2D). In addition, overexpression of LINC00238 significantly decreased the migratory and invasive abilities of Huh7 cells, as determined by Transwell assays ( $\sim 40.0 \%$ inhibition for cell migration and 36.9\% inhibition for cell invasion; Fig. 2E). These in vitro findings suggested that LINC00238 might act as a tumor suppressor in liver cancer.

Knockdown of LINC00238 promotes the liver cancer malignant phenotype in vitro. The expression of LINC00238 was knocked down in HepG2 cells using three shRNAs targeting different sites. The three shRNAs had 82,88 and $72 \%$ knockdown efficiency, respectively (Fig. 3A), and two silencers, shRNA1 and shRNA2, were chosen in subsequent experiments. The CCK- 8 and plate colony formation assay results demonstrated that LINC00238 depletion promoted the viability (Fig. 3B) and colony formation ability of HepG2 cells (Fig. 3C). Furthermore, the Transwell assay results showed that the knockdown of LINC00238 significantly enhanced the migratory and invasive abilities of Hep2 cells compared with the scramble control group (Fig. 3D).

LINC00238 functions as a tumor suppressor in vivo. To further confirm the suppressive effect of LINC00238 in liver cancer in vivo, a modified chick embryo CAM assay was performed to assess tumor growth and metastasis. Control and
LINC00238-overexpressing Huh7 cells labeled with CM-DiI (red fluorescent dye) were inoculated onto CAM to monitor tumor growth and distant metastasis to lung tissues of chick embryos. Compared with the control cells, the overexpression of LINC00238 resulted in a decrease in tumor weight by $64.8 \%$ (Fig. 4A). Moreover, the number of metastatic tumor colonies, which represented metastatic ability to lung tissues, was decreased by $80.9 \%$ in cells after LINC00238 overexpression (Fig. 4B). Consistently, knockdown of LINC00238 expression resulted in a significant increase in not only the tumor weight, but also the extent of metastasis to the lungs (Fig. 4C and D) by CAM assay. Notably, metastatic nodules in lung tissues of the LINC00238 shRNA groups were significantly more abundant than those of the scramble group (Fig. 4D). Hence, the reciprocal effects of LINC00238 overexpression and knockdown both in vitro and in vivo supported the idea that LINC00238 acted as a tumor suppressor in liver cancer.

LINC00238 sponges miR-522. Cytoplasm lncRNAs can regulate cell phenotype by sponging miRNAs (7). The prediction by 1 ncLocator $(30,31)$ (csbio.sjtu.edu.cn/bioinf/lncLocator/) predicted that the cytoplasm locations score of 0.73 , nucleus locations score of 0.03 , ribosome locations score of 0.04 , cytosol locations score of 0.15 and exosome locations score of 0.05 , suggesting that LINC00238 was predominantly located in the cytoplasm. Consistently, nuclear cytoplasmic separation 


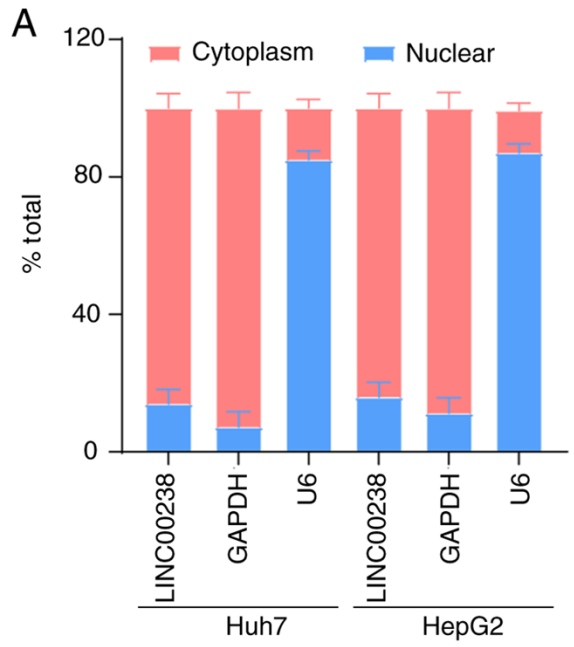

B
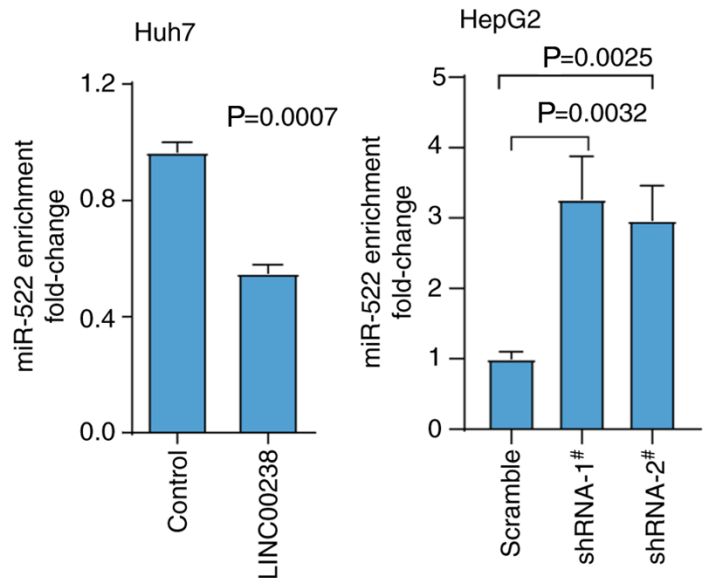

C

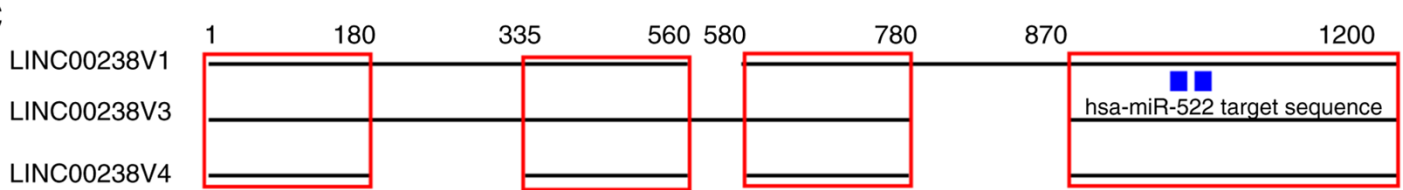

D

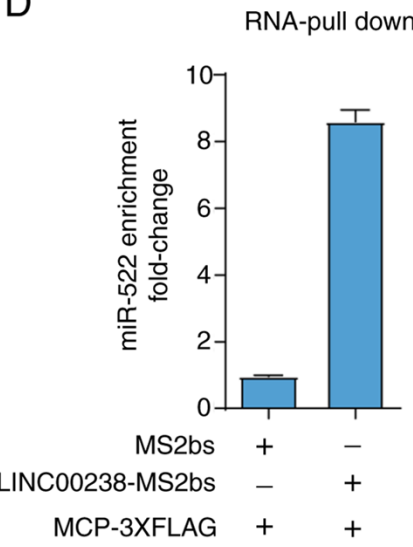

E miR-522-pull down
G

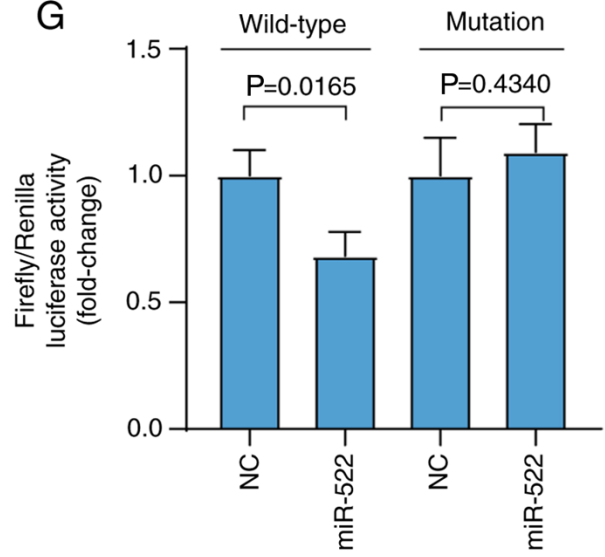

F

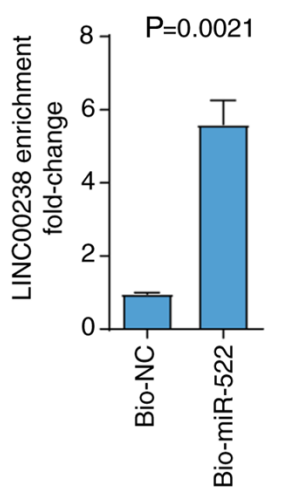
LINC00238V1mut
$5^{\prime}$.......GGCUUUGCCC GCUU
(1084 1090)
LINC00238V1 5'......CGCUCCAUUUGCUU
I I I l l l
522 3' uguGAGAUUUCCCUUGGAAA 5'
(1121 1126)
CCAUUUGT.......3'
|| |||| hsa-miR-522 3' uguGAGAUUUCCCUUGGUAAAa 5'
3' uguGAGAUUUCCCUUGGUAAAa 5'
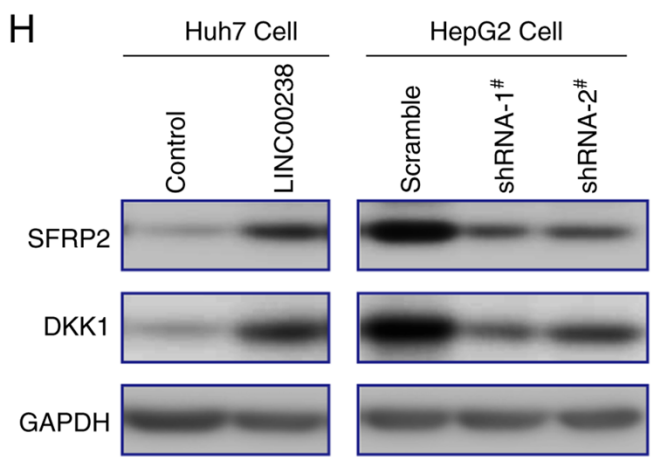

Figure 5. Cytoplasmic LINC00238 sponges miR-522. (A) The bar graphs show that LINC00238 was predominantly localized in cytoplasmic Huh7 and HepG2 cells, as calculated via reverse transcription-quantitative PCR. Cytoplasmic control (GAPDH) and nuclear control (U6) were determined in their expected localization. (B) The expression of miR-522 was downregulated in LINC00238-overexpressing Huh7 cells, but upregulated in LINC00238-knockdown HepG2 cells. (C) Schematic model indicating that three variants of LINC00238 contain the target region of miR-522. (D,E) In vivo and in vitro RNA pull-down assays indicated the interaction between LINC00238 and miR-522. (F) Complementary pairing and corresponding mutation between the sequences of miR-522 and LINC00238. (G) Compared with NC, the miR-522 mimic inhibited the relative fluorescence activity of the LINC00238 wild-type PGL3-control plasmids, but not the mutant type. (H) Western blotting detected that alterations in two known miR-522 targets (SFRP2 and DKK1) were upregulated in LINC00238-overexpressing Huh7 cells and downregulated in LINC00238-knockdown HepG2 cells. miR, microRNA; NC, negative control; SFRP2, secreted frizzled related protein 2; DKK1, dickkopf1; shRNA, short hairpin RNA. 

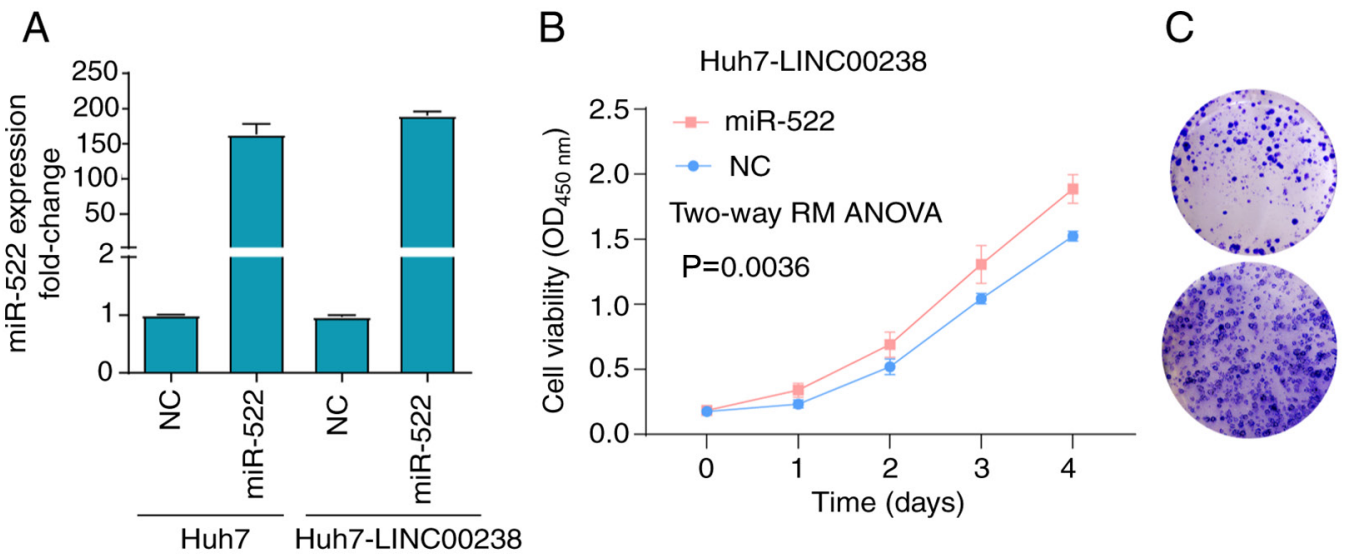

Huh7-LINC00238
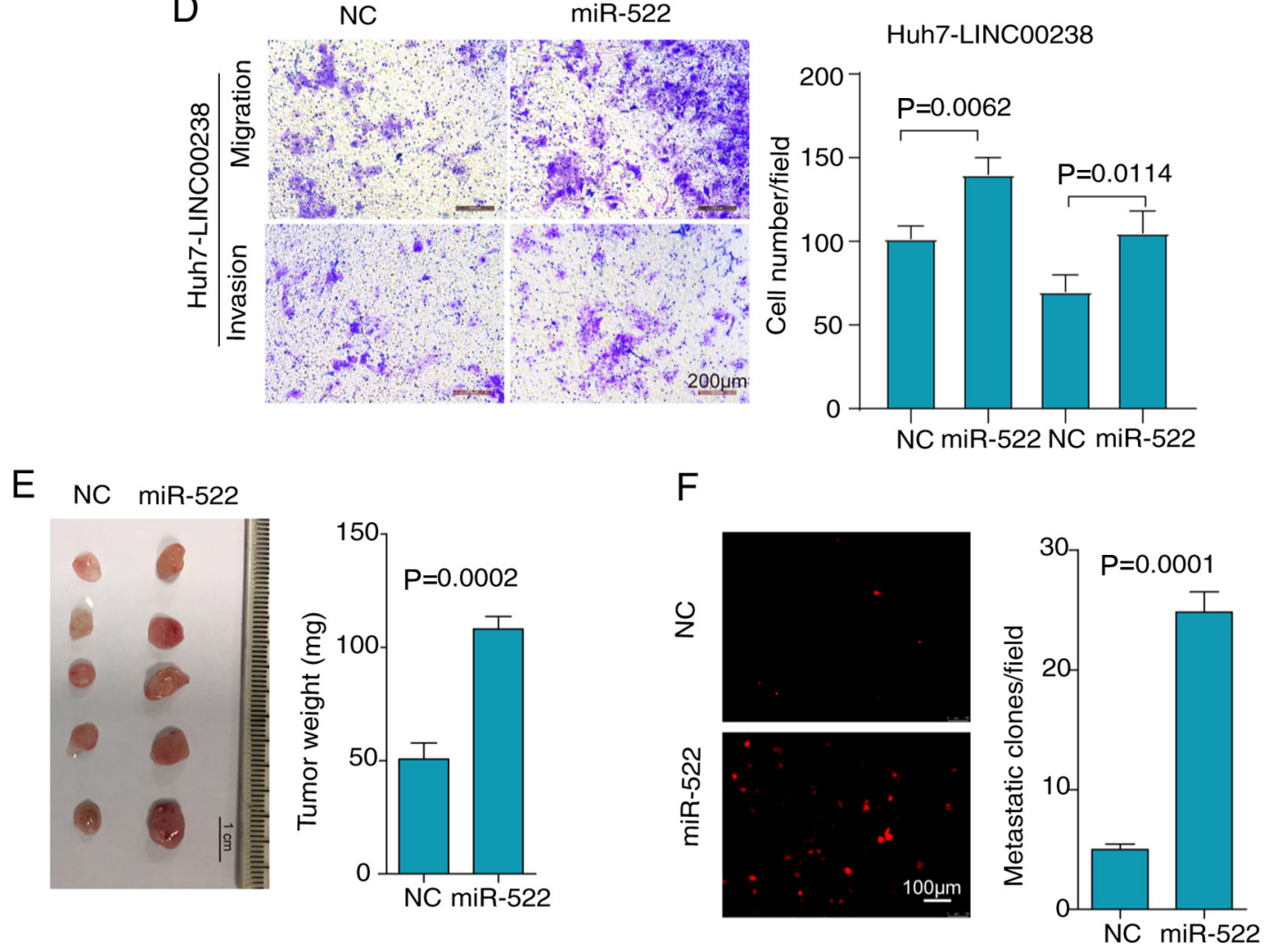

Figure 6. Overexpression of miR-522 partially reverses the suppressive effects of LINC00238. (A) Huh7 cells and LINC00238-overexpressing Huh7 cells were transfected with miR-522 mimics or NC. miR-522 expression was detected via reverse transcription-quantitative PCR. Overexpression of miR-522 in LINC00238-overexpressing Huh7 cells partially reversed the inhibitory effect of LINC00238 on not only (B) cell viability, as determined by Cell Counting Kit-8 assays, (C) cell colony formation, (D) and cell migratory and invasive abilities, as detected by Transwell assays, but also (E) tumor growth and (F) lung metastasis, as determined by the chorioallantoic membrane model. Scale bar, $200 \mu \mathrm{m}$. miR, microRNA; NC, negative control; SFRP2, secreted frizzled related protein 2; DKK1, dickkopf1.

experiments confirmed that LINC00238 was distributed mainly in the cytoplasm (Fig. 5A), which suggested that LINC00238 may sponge miRNAs. Gene Runner software predicted that the target regions 'CCAUUU' of miR-522 were complementary with three isotypes of LINC00238 (Fig. 5C). Furthermore, the expression of miR-522 was decreased in LINC00238-overexpressing Huh7 cells, but increased in LINC00238 knockdown HepG2 cells (Fig. 5B). Then, RNA pull-down experiments suggested that the expression of miR-522 was enriched $~ 8.3$ times by LINC00238 compared with the control group (Fig. 5D). In addition, the expression of LINC00238 in the bio-miR-522 group was $~ 5.8$ times that of the bio-NC group (Fig. 5E). Wild-type and mutant versions of LINC00238 downstream of the luciferase gene were constructed using the PGL3-control vector (Fig. 5F). Using a dual-luciferase reporter assay, overexpression of miR-522 was associated with a decrease in the luciferase activity of the LINC00238 wild-type vector, but had no effect on the mutant vector (Fig. 5G). It was reported previously that miR-522 contributes to the proliferation of liver cancer cells by targeting two Wnt signaling inhibitors, DKK1 and SFRP2 (32). Therefore, it was next investigated whether LINC00238 could suppress the inhibitory effect of miR-522 on these target genes. Consistent with this hypothesis, SFRP2 and 


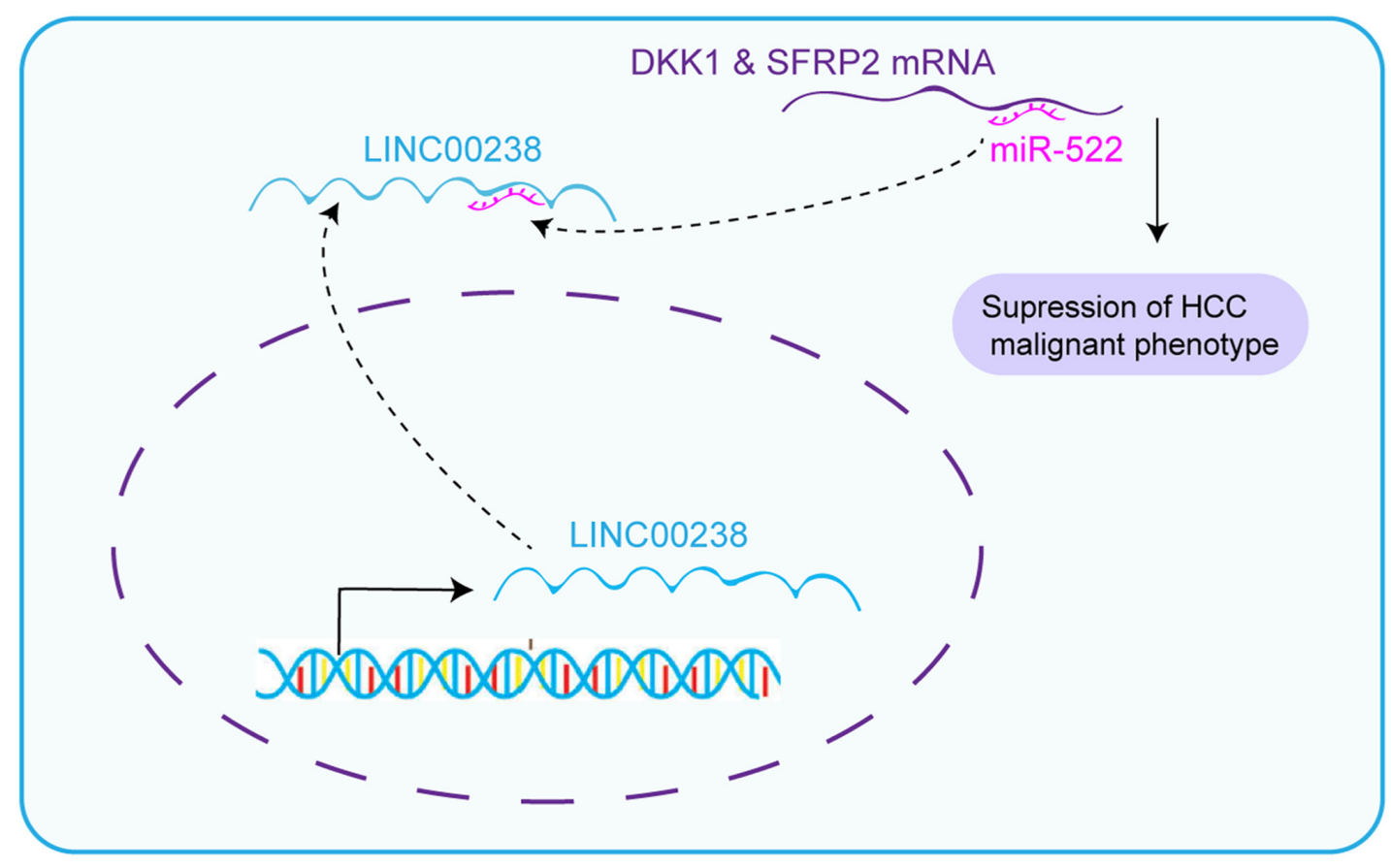

Figure 7. Schematic model for the proposed mechanism of LINC00238. miR, microRNA; SFRP2, secreted frizzled related protein 2; DKK1, dickkopf1; HCC, hepatocellular carcinoma.

DKK1 were increased in Huh7 cell lines after overexpression of LINC00238, but decreased in HepG2 cells after knockdown of LINC00238 (Fig. 5H). Taken together, LINC00238 may regulate the malignant phenotype in liver cancer by sponging miR-522, which leads to release of the inhibition of downstream targets of miR-522.

Overexpression of miR-522 partially reverses the suppressive effects of LINC00238. To corroborate the role of miR-522, a gain-of-function experiment in Huh7-LINC00238 cells was performed by using miR-522 mimics (Fig. 6A). The expression of miR-522 was enhanced 163 -fold and 186-fold after transfection with mimics in Huh7 and Huh7-LINC00238 cells, respectively. Consistent with our prediction and a previous report (32), the overexpression of miR-522 partially reversed the inhibitory effects of LINC00238 on Huh7 cell viability (Fig. 6B), plate colony formation ability (Fig. 6C), migratory and invasive abilities (Fig. 6D), and tumor growth (Fig. 6E) and metastasis to the lungs (Fig. 6F) in the CAM assay. These results confirmed that LINC00238 functioned as a tumor suppressor by sequestering miR-522 at least partially.

\section{Discussion}

In the present study, a suppressive function for LINC00238 in the regulation of the malignant phenotype of liver cancer was proposed. These results suggested that LINC00238 expression was decreased in liver cancer tissues and served as a competing endogenous RNA (ceRNA) that inhibited tumor cell growth and migration via sponging miR-522, which relieved the inhibition of two tumor suppressor targets of miR-522, SFRP2 and DKK1 (Fig. 7).

In the present study, DEGs in liver cancer and normal tissues were assessed by using a web server for cancer and normal gene expression profiling and interactive analyses, GEPIA (11). Among these DEGs, 15 downregulated known lncRNAs were screened out by survival curve analysis, and LINC00238 was selected for further study. Log-rank test supplied by GEPIA was used for the analysis of survival curves. Although there are often obvious violations of the proportional hazard rates due to late-stage crossover, the log-rank test is still used in $70 \%$ of studies (33). Thus, whether the results of the survival curve for lncRNAs where there is late-stage crossover are significant in the current study need further confirmation either by restricting the analyzed period of time to exclude this late crossover event or using a weighted test, such as Renyi or Cramer-von Mises (33). LINC00238 has been reported to be significantly downregulated in liver tissues and liver cells after HBV infection (9). Notably, chronic HBV infection accounts for $\sim 50 \%$ of liver cancer cases worldwide (28). Recently, LINC00238 has been reported in liver cancer as a tumor suppressor by activating the apoptosis pathway (29). However, further experiments, including in vivo assays, are still required to support the suppressive functional role of LINC00238, and determine its subcellular localization and underlying mechanisms. In the current study, consistent with a previous report (29), the in vitro experiments validated LINC00238 as a tumor suppressor. In addition, its tumor suppressor role was further validated by in vivo experiments and a novel mechanism as a ceRNA via the sponging miR-522 was reported. Although LINC00238 was confirmed as a predictor of clinical prognosis from the databank, further studies are needed to confirm LINC00238 as a clinical predictor from our own cohort of patients. A considerable number of studies have examined whether lncRNAs, either as oncogenes or suppressor genes, function as ceRNAs in liver cancer. For example, HOXD-AS1, as an oncogene, sponges regulatory miR-130a-3p to enhance the expression of the transcription factor SOX4, thus resulting in the promotion 
of liver cancer metastasis (34). LncRNA CASC2, a novel tumor suppressor, exerts antimetastatic effects through the miR-367/FBXW7 axis in liver cancer cells (35). Previous work suggests that lncRNAs in the cytoplasm function as molecular sponges or modulate mRNA stability (6). In the present study, LINC00238 was identified to be located in the cytoplasm and considered to be a ceRNA that regulates liver cancer progression. The RNA binding immunoprecipitation assay suggested that LINC00238 had the potential to interact with miR-522. Dual luciferase reporter and biotin-miR-522 pull-down assays also revealed that LINC00238 was a target of miR-522. It is commonly known that miR-522 is an oncogene in a number of cancers, including liver (36) and lung (37) cancer. SFRP2 and DKK1, two known Wnt signaling inhibitors involved in $\mathrm{HCV}$-induced multistep hepatocarcinogenesis (38), have already been identified as two direct targets of miR-522 in liver cancer cells (32). In the current study, although it was demonstrated that LINC00238 sponged miR-522, and that the expression of miR-522 was decreased in LINC00238-overexpression Huh7 cells and increased in LINC00238-knockdown HepG2 cells, further study to determine the associations between the expression levels of LINC00238 and miR-522 in patients with liver cancer are required.

In summary, it was speculated that the suppressive effects of LINC00238 on the liver cancer malignant phenotype may be due to miR-522-mediated regulation of SFRP 2 and DKK1. The ceRNA regulatory network (LINC00238/miR-522/SFRP2 and DKK1) shed light on the mechanisms of lncRNA regulation of liver cancer development. It is worth noting that LINC00238 may also regulate liver cancer progression through other mechanisms, such as the regulation of mRNA stability.

\section{Acknowledgements}

Not applicable.

\section{Funding}

No funding was received.

\section{Availability of data and materials}

The datasets used and/or analyzed during the current study are available from the corresponding author on reasonable request.

\section{Authors' contributions}

HQ and $\mathrm{CH}$ designed and performed the research, wrote the manuscript and confirm the authenticity of all the raw data. QW analyzed the data from The Cancer Genome Atlas. JW, XT and WX analyzed the data. All authors have read and approved the final manuscript.

\section{Ethics approval and consent to participate}

Not applicable.

\section{Patient consent for publication}

Not applicable.

\section{Competing interests}

The authors declare that they have no competing interests.

\section{References}

1. Siegel RL, Miller KD and Jemal A: Cancer statistics, 2019. CA Cancer J Clin 69: 7-34, 2019.

2. Chen W, Zheng R, Baade PD, Zhang S, Zeng H, Bray F, Jemal A, Yu XQ and He J: Cancer statistics in China, 2015. CA Cancer J Clin 66: 115-132, 2016.

3. Anwanwan D, Singh SK, Singh S, Saikam V and Singh R: Challenges in liver cancer and possible treatment approaches. Biochim Biophys Acta Rev Cancer 1873: 188314, 2020.

4. Mikhail S, Cosgrove D and Zeidan A: Hepatocellular carcinoma: Systemic therapies and future perspectives. Expert Rev Anticancer Ther 14: 1205-1218, 2014.

5. Jarroux J, Morillon A and Pinskaya M: History, discovery, and classification of lncRNAs. Adv Exp Med Biol 1008: 1-46, 2017.

6. Fatica A and Bozzoni I: Long non-coding RNAs: New players in cell differentiation and development. Nat Rev Genet 15: 7-21, 2014.

7. Chen LL: Linking long noncoding RNA localization and function. Trends Biochem Sci 41: 761-772, 2016.

8. Lim LJ, Wong SYS, Huang F, Lim S, Chong SS, Ooi LL, Kon OL and Lee CG: Roles and regulation of long noncoding RNAs in hepatocellular carcinoma. Cancer Res 79: 5131-5139, 2019.

9. Qin H, Zhenzhen Z and Quanbo L: Effect of long intergenic non-coding RNA 238 on HBV replication and expression in vitro. J Third Military Med Univ 40: 1942-1947, 2018.

10. Gao Q, Zhu H, Dong L, Shi W, Chen R, Song Z, Huang C, Li J, Dong X, Zhou Y, et al: Integrated Proteogenomic characterization of HBV-related hepatocellular carcinoma. Cell 179: 561-577. e22, 2019.

11. Tang Z, Li C, Kang B, Gao G, Li C and Zhang Z: GEPIA: A web server for cancer and normal gene expression profiling and interactive analyses. Nucleic Acids Res 45: W98-W102, 2017.

12. Ribatti D: The Chick Embryo Chorioallantoic Membrane in the Study of Angiogenesis and Metastasis. Springer, Netherlands, 2010.

13. Pinto MT, Ribeiro AS, Conde I, Carvalho R and Paredes J: The chick chorioallantoic membrane model: A new in vivo tool to evaluate breast cancer stem cell activity. Int J Mol Sci 22: 334 , 2020.

14. Zabielska K, Lechowski R, Król M, Pawłowski KM, Motyl T, Dolka I and Zbikowski A: Derivation of feline vaccineassociated fibrosarcoma cell line and its growth on chick embryo chorioallantoic membrane-a new in vivo model for veterinary oncological studies. Vet Res Commun 36: 227-233, 2012.

15. Cimpean AM, Lalosevic D, Lalosevic V, Banovic P, Raica M and Mederle OA: Disodium Cromolyn and Anti-podoplanin antibodies strongly inhibit growth of BHK 21/C13-derived Fibrosarcoma in a chick Embryo chorioallantoic membrane model. In Vivo 32: 791-798, 2018.

16. Klingenberg M, Becker J, Eberth S, Kube D and Wilting J: The chick chorioallantoic membrane as an in vivo xenograft model for Burkitt lymphoma. BMC Cancer 14: 339, 2014.

17. Li M, Pathak RR, Lopez-Rivera E, Friedman SL, Aguirre-Ghiso JA and Sikora AG: The In Ovo chick chorioallantoic membrane (CAM) assay as an efficient Xenograft model of hepatocellular carcinoma. J Vis Exp: 52411, 2015 doi: $10.3791 / 52411$.

18. Avram S, Coricovac DE, Pavel IZ, Pinzaru I, Ghiulai R, Baderca F, Soica C, Muntean D, Branisteanu DE, Spandidos DA, et al: Standardization of A375 human melanoma models on chicken embryo chorioallantoic membrane and Balb/c nude mice. Oncol Rep 38: 89-99, 2017.

19. Wilson SM and Chambers AF: Experimental metastasis assays in the chick embryo. Curr Protoc Cell Biol Chapter 19: Unit 19.6, 2004.

20. Livak KJ and Schmittgen TD: Analysis of relative gene expression data using real-time quantitative PCR and the 2(-Delta Delta C(T)) method. Methods 25: 402-408, 2001.

21. Dash S, Balasubramaniam M, Dash $\mathrm{C}$ and Pandhare J: Biotin-based Pulldown assay to validate mRNA targets of cellular miRNAs. J Vis Exp: 57786, 2018 doi: 10.3791/57786. 
22. Zheng YL, Li L, Jia YX, Zhang BZ, Li JC, Zhu YH, Li MQ, He JZ, Zeng TT, Ban XJ, et al: LINC01554-mediated glucose metabolism reprogramming suppresses tumorigenicity in hepatocellular carcinoma via downregulating PKM2 expression and inhibiting Akt/mTOR signaling pathway. Theranostics 9: 796-810, 2019.

23. Ding Y, Sun Z, Zhang S, Chen Y, Zhou B, Li G, Sun Q, Zhou D, Ge Y, Yan S and Wang W: Down-regulation of Long Non-coding RNA LINC01554 in hepatocellular cancer and its clinical significance. J Cancer 11: 3369-3374, 2020.

24. He J, Zuo Q, Hu B, Jin H, Wang C, Cheng Z, Deng X, Yang C, Ruan $\mathrm{H}, \mathrm{Yu} \mathrm{C}$, et al: A novel, liver-specific long noncoding RNA LINC01093 suppresses HCC progression by interaction with IGF2BP1 to facilitate decay of GLI1 mRNA. Cancer Lett 450: 98-109, 2019.

25. Zheng Y, Yu K, Huang C, Liu L, Zhao H, Huo $M$ and Zhang J: Integrated bioinformatics analysis reveals role of the LINC01093/miR-96-5p/ZFAND5/NF- $\mathrm{B}$ signaling axis in hepatocellular carcinoma. Exp Ther Med 18: 3853-3860, 2019.

26. Wang S, Xu M, Sun Z, Yu X, Deng Y and Chang H: LINC01018 confers a novel tumor suppressor role in hepatocellular carcinoma through sponging microRNA-182-5p. Am J Physiol Gastrointest Liver Physiol 317: G116-G126, 2019.

27. Wen Q, Wang D, Yang Y, Chen X, Pan X, Han Q, Deng Y, Li X, Chen X, Yan J and Zhou J: Competing endogenous RNA screening based on long noncoding RNA-messenger RNA co-expression profile in Hepatitis B virus-associated hepatocarcinogenesis. J Tradit Chin Med 37: 510-521, 2017.

28. Xie Y: Hepatitis B Virus-associated hepatocellular carcinoma. Adv Exp Med Biol 1018: 11-21, 2017.

29. Jiang C, Li F, Yang M, Duan J, Lai J, Sun S and Fan S: LINC00238 inhibits hepatic carcinoma progression by activating TMEM106C-mediated apoptosis pathway. Mol Med Rep 24: 757, 2021.

30. Lin Y, Pan X and Shen HB: 1ncLocator 2.0: A cell-line-specific subcellular localization predictor for long non-coding RNAs with interpretable deep learning. Bioinformatics 37: 2308-2316, 2021.
31. Cao Z, Pan X, Yang Y, Huang Y and Shen HB: The lncLocator: A subcellular localization predictor for long non-coding RNAs based on a stacked ensemble classifier. Bioinformatics 34: 2185-2194, 2018

32. Zhang H, Yu C, Chen M, Li Z, Tian S, Jiang J and Sun C: miR-522 contributes to cell proliferation of hepatocellular carcinoma by targeting DKK1 and SFRP2. Tumour Biol 37: 11321-11329, 2016.

33. Li H, Han D, Hou Y, Chen H and Chen Z: Statistical inference methods for two crossing survival curves: A comparison of methods. PLoS One 10: e0116774, 2015.

34. Wang H, Huo X, Yang XR, He J, Cheng L, Wang N, Deng X, Jin $\mathrm{H}$, Wang N, Wang C, et al: STAT3-mediated upregulation of lncRNA HOXD-AS1 as a ceRNA facilitates liver cancer metastasis by regulating SOX4. Mol Cancer 16: 136, 2017.

35. Wang Y, Liu Z, Yao B, Li Q, Wang L, Wang C, Dou C, Xu M, Liu Q and Tu K: Long non-coding RNA CASC2 suppresses epithelial-mesenchymal transition of hepatocellular carcinoma cells through CASC2/miR-367/FBXW7 axis. Mol Cancer 16: 123,2017

36. Shi YH, Qi BB, Liu XB and Ding HM: Upregulation of miR-522 is associated with poor outcome of hepatocellular carcinoma. Eur Rev Med Pharmacol Sci 20: 3194-3198, 2016.

37. Zhang T, Hu Y, Ju J, Hou L, Li Z, Xiao D, Li Y, Yao J, Wang C, Zhang Y and Zhang L: Downregulation of miR-522 suppresses proliferation and metastasis of non-small cell lung cancer cells by directly targeting DENN/MADD domain containing 2D. Sci Rep 6: 19346, 2016.

38. Umer M, Qureshi SA, Hashmi ZY, Raza A, Ahmad J, Rahman M and Iqbal M: Promoter hypermethylation of Wnt pathway inhibitors in hepatitis $\mathrm{C}$ virus-induced multistep hepatocarcinogenesis. Virol J 11: 117, 2014.

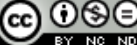

This work is licensed under a Creative Commons Attribution-NonCommercial-NoDerivatives 4.0 International (CC BY-NC-ND 4.0) License. 